\title{
Intestinal Crypts Reproducibly Expand in Culture
}

\author{
Megan K. Fuller, M.D. ${ }^{1,{ }^{*},}$ Denver M. Faulk, B.S. ${ }^{2,{ }^{*}}$, Nambirajan Sundaram, Ph.D. ${ }^{2}$, Noah F. \\ Shroyer, Ph.D. ${ }^{3}$, Susan J. Henning, Ph.D. ${ }^{4}$, and Michael A. Helmrath, M.D. ${ }^{2}$ \\ ${ }^{1}$ Department of Surgery, University of North Carolina at Chapel Hill \\ ${ }^{2}$ Department of Surgery, Cincinnati Children's Hospital Medical Center. \\ ${ }^{3}$ Department of Gastroenterology, Cincinnati Children's Hospital Medical Center. \\ ${ }^{4}$ Department of Medicine and Cellular \& Molecular Physiology, University of North Carolina at \\ Chapel Hill
}

\begin{abstract}
Background-In vitro growth techniques for intestinal crypts and single intestinal stem cells have been recently described, but several questions of translational importance remain unaddressed. The purpose of this study was to: first, evaluate if intestinal crypts reproducibly expand in vitro; second, determine the impact of age and region of intestine on crypt growth in vitro; and third, determine the effects of cryopreservation on crypt growth in vitro.
\end{abstract}

Methods and Materials-Crypts were harvested, from $5 \mathrm{~cm}$ of proximal, middle and distal small intestine of C57BL/6J mice aged 4wk, 6-8wk, 12-14wk, and 18-20wk ( $\mathrm{n}=4-6$ animals), and cultured. For each region, we determined the efficiency of crypts forming enterospheres (Day 1), and progressing to enteroids (Day 7). Subsequently, enteroids were passaged and cryopreserved to determine if growth was changed by these manipulations.

Results-43-99\% of intestinal crypts formed enterospheres, with higher efficiency in proximal small intestine and in younger mice. 25-64\% of enterospheres progressed to budding enteroids within 7 days. In vitro expansion was greater in proximal enteroids. This expansion continued in a logarithmic fashion, with $\sim 97 \%$ replating efficiency of isolated enteroid crypt buds. Following cryopreservation, $\sim 90 \%$ of enteroids recovered normal proliferative capacity.

Conclusions-Intestinal crypt culture is efficient and significantly expands intestinal tissue in a reproducible manner. Regional and age growth differences may reflect distinct stem cell characteristics or differences in support cells. The ability to culture and expand intestinal tissue in vitro provides a potential translational approach toward understanding and treating patients with short bowel syndrome.

\section{Keywords}

small intestine; enteroid; organoid; crypt; in vitro; intestinal stem cell

\footnotetext{
(C) 2012 Elsevier Inc. All rights reserved.

Corresponding Author: Michael A. Helmrath, MD, Department of Surgery, Division of Pediatric Surgery, Cincinnati Children's Hospital Medical Center, 3333 Burnet Avenue, MLC 2023, Cincinnati, OH 45229-3039. michael.helmrath@ cchmc.org; phone: 513-803-7330; fax: 513-636-3248 (current address).

*Both authors contributed equally to this work

Publisher's Disclaimer: This is a PDF file of an unedited manuscript that has been accepted for publication. As a service to our customers we are providing this early version of the manuscript. The manuscript will undergo copyediting, typesetting, and review of the resulting proof before it is published in its final citable form. Please note that during the production process errors may be discovered which could affect the content, and all legal disclaimers that apply to the journal pertain.

Disclosures: The authors have no conflicts to disclose.
} 


\section{Introduction}

Short bowel syndrome (SBS) occurs when loss of intestinal length results in the inability of the bowel to absorb the nutrients and/or fluids necessary for normal physiology and growth[1]. Current therapy for SBS supports fluid and nutritional needs in the form of parenteral nutrition (PN) that is gradually weaned with concomitant increases in enteral nutrition over the period of time required for the remaining intestine to adapt. When adequate adaptation does not occur within 2-3 years following intestinal injury or loss, a lifetime requirement for $\mathrm{PN}$ is common. Current literature indicates that the preserved region of bowel affects outcomes, as patients without their distal ileum and proximal colon require longer periods of time to adapt and wean off of PN. These patients also have a higher incidence of chronic intestinal failure compared to patients with similar losses of proximal jejunum [2]. Supporting this finding, several studies demonstrate a greater ability of the ileum to undergo hyperplasia and adaptive response following resection of the proximal bowel than the converse [3]. This regional disparity in adaptation may be due to differences in the intestinal stem cells that continuously regenerate the epithelium.

Ideally, the use of tissue-engineered small intestine (TESI) from ex-vivo expansion of a patient's own tissue offers therapeutic potential for SBS by either increasing overall absorptive surface area or specifically restoring the physiological loss of regionalized bowel. TESI investigators have demonstrated proof of principle by intestinal formation in large animal models, and abrogation of nutritional deficits in a small animal model of SBS [4-6]. Despite this progress, there remain limitations, namely: (a) the reliance on neonatal donor tissue; and (b) the inability to expand the donor tissue [7].

Early ex vivo/ in vitro culture methods of the small bowel are marked by numerous drawbacks including lack of normal morphology, degradation with time, and lack of appropriate differentiation [8]. However more recently, long-term culture conditions, which differentiate and recapitulate normal crypt-villus architecture have been devised initially for mouse tissue $[9,10]$ and subsequently for human tissue [9, 11-13]. These studies also suggest an expansion of starting tissue [9-13].

To date, despite these advances in culture of the small intestine there has been no systematic and quantitative assessment of the capacity to expand tissue either in vitro or in vivo as required for TESI to be clinically practical [7]. In fact, with current methods there is a substantial loss of tissue, limiting the current usefulness for therapy. Thus, with recent advances in methods for intestinal epithelial culture, we sought to answer three questions: (1) Does age impact the ability to efficiently culture crypts across adulthood; (2) Does the region of origin impact the ability to efficiently culture crypts; (3) Can we expand and store isolated intestinal crypts with current culture techniques?

\section{Materials and Methods}

\section{Experimental Design}

To investigate the influence of a) age and b) region, on crypt culture in a quantitative fashion, we separated the small intestine into three regions termed proximal, middle, and distal. We further categorized mice by age to cover weanlings to adulthood. We then cultured crypts isolated from these categories and monitored their progress. To evaluate the ability of crypts to expand in vitro we examined the number of new crypt domains and maintenance of budding over time. To evaluate the ability to store expanded crypts for later usage, we examined percent of enteroids revived after freezing and maintenance of crypt budding after freeze-thaw. 


\section{Animals}

All animal procedures were approved by the Institutional Animal Care and Use Committee at UNC and CCHMC. Male, C57BL/6J mice were obtained from Jackson Laboratories (Bar Harbor, ME) at ages 4, 6-8, 12-14, and 18-20 weeks of age $(n=4,6,4,6)$. They were subsequently housed four per cage on a $12 \mathrm{hr}$ light dark cycle.

\section{Tissue Harvest for Intestinal Crypts}

After carbon dioxide euthanasia, the small intestine was removed and flushed with cold phosphate buffered saline (PBS), the first 2-3 cm adjacent to the pylorus and the distal 2-3 $\mathrm{cm}$ just proximal to the ileocecal valve were discarded. The $5 \mathrm{~cm}$ adjacent to these regions were termed "proximal" and "distal" respectively. The "middle" region was defined as the 5 $\mathrm{cm}$ at the midpoint of the remaining intestine. Each segment, proximal (P), middle (M), or distal (D), was then opened longitudinally and cut into $5 \mathrm{~mm}$ pieces. Pieces were transferred to $3 \mathrm{mM}$ EDTA in PBS and rocked for $30 \mathrm{~min}$ at $4^{\circ} \mathrm{C}$. After manual shaking for $30 \mathrm{sec}$, the tissue was moved to fresh cold PBS and shaken for 3-5 min. Sorbitol (2\%) (SIGMA, St. Louis, MO) was then added 1:1 to the solution and the crypts filtered through a $70 \mu \mathrm{m}$ filter. The flow-through was then checked for crypt density and an appropriate volume pelleted at $150 \mathrm{xg}$ for $10 \mathrm{~min}$ at $4^{\circ} \mathrm{C}$.

\section{Crypt Culture and Quantification}

The pelleted crypts were resuspended in growth factor reduced Matrigel (BD Bioscience) supplemented with EGF (50ng/mL), Noggin (100ng/mL), R-spondin $(500 \mathrm{ng} / \mathrm{mL})$, wnt3a $(5 \mathrm{ng} / \mathrm{mL})$, and Y27632 $(10 \mu \mathrm{M})$ (R\&D Systems, Minneapolis, MN; Invitrogen, Carlsbad, CA). [All concentrations are for total well volume.] With the exception of wnt3a, these conditions are those described by Sato et al [14]. A mean of 29 crypts in $10 \mu \mathrm{L}$ of Matrigel were then plated in 6 experimental replicates per region on 48 well plates. Each was then overlaid with DMEM/F12 (GIBCO, Carlsbad, CA) supplemented with Hepes (10mM), N2 (1:100), B27 (1:50), L-glutamine (1:100), and penicillin/streptomycin (1:100) (Invitrogen).

The final number of crypts plated was counted per well. Each well was quantified on day 1 of culture for enterosphere (Fig. 1C) formation. At Day 4, media was changed and EGF, Noggin, R-spondin, and wnt3a at original concentrations were added to each well. At Day 7, each well was counted for enteroid formation (Fig. 1D). Among formed enteroids, lateral crypt buds were counted across a minimum of two wells and ten enteroids per region and mouse. Enterosphere and enteroid efficiencies were defined as a percentage of enterospheres or enteroids, respectively, divided by the number of crypts initially plated.

\section{Maintenance of Enteroids over Time}

To assess the performance of cultures over time and promote continued growth, enteroids were mechanically dissociated via passage through a 30 gauge needle after 7 days in culture. Enteroid units were then resuspended in PBS and pelleted at 150xg. Supernatant of PBS and Matrigel was discarded and enteroid units resuspended in fresh Matrigel according to initial crypt culture conditions. This was repeated every 7 days for the life of the culture. For quantification, only passages resulting in single crypt buds were utilized. Number of crypt buds replated was counted on day 1 after passaging. The number of enteroids formed from these buds was then counted on day 7 prior to the next passage. Quantification of the $1^{\text {st }}$ and $2^{\text {nd }}$ passages ( 21 days in culture) was performed in 4 experimental replicates per mouse.

\section{Effect of Freeze-Thaw}

Enteroids were cultured as above and counted then passaged at 7 days. After an additional 3 days in culture, enteroids were removed from Matrigel by aspirating media off each well, 
adding 500uL of PBS, and repeatedly pipetting to breakup Matrigel. The contents of each well were removed and $5 \mathrm{~mL}$ of PBS added then centrifuged for $10 \mathrm{~min}$ at $150 \mathrm{xg}$. The supernatant was discarded and enteroids were then resuspended in freezing media (advanced DMEM/F12 with 10\%DMSO and 10\% FBS). Enteroids were then frozen in an isopropyl alcohol freezing container (Nalgene, Rochester, NY). After freezing, vials were transferred to liquid N2 storage. After 7 days in storage, enteroids were quickly thawed then centrifuged for $10 \mathrm{~min}$ at $150 \mathrm{xg}$ to remove the freezing media. Enteroids were resuspended in Matrigel with growth factors and overlaid with media as previously described. At 1 day in culture, enteroids were counted for initial plating density per well. After 3 days in culture, enteroids were assessed for viability based on morphology. Enteroids were then passaged and plated at a mean density of 25 buds/well. These buds were then cultured an additional 7 days before crypt budding was assessed as previously described.

\section{Statistical Analysis}

To analyze progression of: crypts to enterospheres, enterospheres to enteroids, and enterospheres to enteroids by age and region, three repeated measures logistic regression analyses were performed. The response variable was yes/no on: 1) whether a crypt survived to an enterosphere at day 1, (2) whether a crypt progressed to an enteroid at day 7, and (3) whether an enterosphere at day 1 progressed to an enteroid at day 7 . For each analysis, the site factor (P, M, and D) was nested within the time factors (weeks 4, 8, 14, and 20). Since measurements within the same mouse at the same site were expected to have similarly correlated measurements a compound symmetric correlation matrix was used. An overall test of interaction between site and time was performed and if it proved statistically significant then subgroup comparisons of interest were made. Additionally, to predict bud counts a Poisson regression was performed in an otherwise similar manner to aforementioned models. P-values less than 0.05 were considered statistically significant. Analyses were performed using SAS Version 9.2 (SAS Institute, Cary NC).

To analyze the maintenance of efficiency over time and crypt budding over time in culture, as a single region and time were utilized, 1-way ANOVA with Bonferroni posttest corrections for multiple comparisons was performed. P-values less than 0.05 were considered significant. Analyses were performed in GraphPad Prism 5 (LaJolla, CA).

\section{RESULTS}

\section{Behavior of Crypts in Culture}

As shown in Fig. 1A-B, following plating, crypts rapidly move from normal crypt architecture to formation of spheroid structures. In accordance with the recommendations from the Intestinal Stem Cell Consortium (ISCC http://iscc.coh.org), we have termed these structures "enterospheres." Over subsequent time in culture the enterospheres undergo extensive budding to give rise to more complex structures termed "enteroids" (Fig. 1C). A typical enteroid from the proximal intestine at 7 days in culture is shown in Fig. 1D. The term "organoids" will be reserved for cultures also containing mesenchyme (ISCC http:// iscc.coh.org).

\section{Efficiency of Enterosphere Formation}

Tissue from all regions of the small intestine and from all ages studied formed enterospheres with mean efficiencies ranging from 43-99\% of crypts plated (Fig. 2A). Both age and region demonstrated a significant effect on survival and significantly interacted with each other ( $p$ $<0.02$ ). As such, subgroup analyses were performed within age or region. Efficiency was significantly greater in the proximal than the distal segment at 14 and $20 \mathrm{wks}(\mathrm{p}<0.05)$. At 4 wks of age, there was a trend to greater efficiency in the proximal than the distal segment. 
At 8 wks of age, all segments formed enterospheres equally well. Within all regions, 20wk crypts formed enterospheres less efficiently than $14 \mathrm{wk}(\mathrm{p}<0.05)$. In the distal and middle regions, 20 wks was also less efficient than $8 \mathrm{wk}(\mathrm{p}<0.05)$.

\section{Efficiency of Enteroid Formation}

Tissue from all regions of the small intestine and ages studies formed enteroids with mean efficiencies ranging from $25-64 \%$ of crypts plated (Fig. 2B). Significant interaction persisted between age and region ( $\mathrm{p}<0.02$ ). In contrast to enterosphere efficiency, enteroids formed equally well between regions at 4,8 , and 14 wks. Only at 20 wks was the proximal region more efficient than middle or distal regions at enteroid formation $(\mathrm{p}<0.05)$. However in examining animal age, within all regions $8 \mathrm{wk}$ crypts were more likely to form enteroids than 14 or $20 \mathrm{wk}(\mathrm{p}<0.05)$.

\section{Progression from Enterosphere to Enteroid}

We next calculated progression of enterospheres to enteroids to determine if low enteroid efficiency was caused by failure of enterosphere formation or failure of progress once initially formed. Progression occurred in $29-76 \%$ of enterospheres (Fig. 2C).

Enterospheres progressed equally well between regions at each age. However, 8 wks of age progressed significantly better than 14 wks across all regions $(\mathrm{p}<0.05)$. Within the proximal crypts, $20 \mathrm{wks}$ progressed significantly better than $14 \mathrm{wks}(\mathrm{p}<0.05)$.

\section{Number of Crypt Buds per Enteroid}

To assess regional and age influences on in vitro growth, the number of crypt buds per enteroid was quantified as a surrogate for expansion of intestinal stem cells, as each bud is capable of independently forming a new enteroid. No significant interaction was present between age and region ( $p>0.12$ ); thus, main effects of age and region were compared. At 4 wks of age enteroids possessed significantly fewer buds than 8,14 , or 20 wks, which did not differ from each other ( $\mathrm{p}<0.005$ data not shown). As shown in Fig. 3, a strong regional effect was present with number of crypt buds greatest proximally (7.2) and declining through the middle (5.3) and distal (3.7) regions ( $\mathrm{p}<0.001)$.

\section{Passaging of Enteroids to Determine Efficiency and Proliferation Over Time}

Although we initially confirmed expansion of crypts, we further examined the potential to expand crypts in vitro through passaging of enteroids to promote continued growth. Enteroids passaged down to single crypt buds were replated. There was high efficiency ( $93-$ $100 \%$ ) of crypt buds progressing to new enteroids with passaging (Fig. 4). Additionally, enteroids maintained their ability for continued expansion, as there is no significant difference in the number of crypts buds formed per enteroid in reference to initially plated crypts forming enteroids at day 7(data not shown).

\section{Enteroid Efficiency Following Freeze-Thaw}

Following thawing of enteroids, $90 \% \pm 2$ and $87 \% \pm 2$ survival was observed for proximal and distal enteroids respectively $(n=4)$. In addition, enteroid morphology and crypt budding was maintained as prior to freezing (data not shown).

\section{Fold Expansion}

The ability of crypts to be expanded for autotransplantation is one of the major goals of in vitro culture; as such, fold expansion was calculated. As each crypt bud represents a new crypt domain, fold expansion was calculated by multiplying enteroid efficiency at 7 days by the mean number of crypt buds per enteroid. Pooled data for all ages showed that expansion varied between 3.4 to 1.4 fold at 7 days from proximal to distal respectively (Fig. 5). If the 
single most proliferative age and region is examined, $8 \mathrm{wks}$ of age in the proximal intestine, expansion at 7 days is already 4 fold (Fig. 5). If we take this culture out to 28 days and 2 passages, each dissociated crypt bud will reform an enteroid with an average of 7 buds. Thus, we will go from 4 crypts to 28 crypts after passage 1 (100\% efficiency). These 28 enteroids will passage with $93 \%$ efficiency to form to 182 crypts. When passaged at $93 \%$ efficiency, these 182 enteroids will represent 1183 new crypt axes at 28 days in culture. We have continued this process with similar efficiency beyond 10 passages.

\section{Discussion}

While culture techniques to maintain ISC and intestinal crypts have been recently described, several issues of translational importance had not been previously investigated. In this study, we have demonstrated that: a) crypt culture is influenced by both the age and region of the donor small intestine; b) crypts can be expanded in vitro; c) expanded crypts can be stored for later usage.

On examination of the effects of age and region on crypt culture, overall there is good progression across ages and regions examined. However, the proximal jejunum tended to form enteroids more efficiently than the distal ileum. Interestingly, this regional effect may be secondary to the ability of proximal crypts to initially form enterospheres more efficiently, as once enterospheres are formed there is no significant difference in their progression to enteroids. Likewise, $20 \mathrm{wk}$ adult crypts were less likely to form enterospheres, but once formed enterospheres progressed well and in fact better than at 14 wks of age. Assuming the behavior of mouse tissue predicts that of human tissue, this implies that translational applications will not be limited to embryonic and fetal sources of intestine; autotransplantation, even into adulthood, may be possible by expansion of the differentiated stem cells present among crypts for all regions of the bowel.

In the initial report of crypt culture Sato et al. presented convincing evidence that enteroid crypt buds represent new crypt-villus axes [14]. As such, enteroid budding may be akin to expansion of the intestinal stem cell (ISC) compartment and formation of new crypts via crypt fission [15]. On close examination of our data related to crypt budding, a marked proximal (highest) to distal (lowest) gradient of bud number was observed. This implies that the ISCs of the proximal intestine may be either more responsive to culture conditions (which are high in wnt signals), or may possess intrinsic differences to ISCs of the distal intestine. Alternatively the support cells at the crypt base may function differently in the proximal intestine than in the distal intestine. Isografts with intact fetal intestine have demonstrated maintenance of regional expression [16]; however, recent culture systems have yet to examine whether this holds true in vitro. These differences in proximal-distal crypt budding, suggest that the functional diversity of small intestine in vivo may be maintained in vitro. This also underscores the potential ability of this robust culture system to be used for asking questions of the crypt-villus axis and the signaling involved in normal homeostasis and in disease states.

One of the most important findings of this paper is that under these culture conditions crypts can be significantly expanded in vitro. As crypt buds represent new crypt-villus axes they are also indicative of tissue expansion in vitro. If anything, this study underestimates crypts potential for expansion by only counting lateral buds. Nonetheless, expansion at 7 days in culture was observed for even the distal region, which exhibited the least efficiency of enteroid formation. Furthermore, each enteroid, when dissociated to single crypt buds, retains its proliferative capacity so with increased time in culture tissue will expand logarithmically. Additionally, there is little loss of enteroids following cryopreservation and enteroids maintain their proliferative capacity after thawing. These abilities to expand tissue 
in vitro, store it, and subsequently utilize it holds tremendous promise for future therapeutic usage.

Gut tissue engineering has become increasingly sophisticated, with the ability for anastomosis and abrogation of intestinal failure; however, as noted in Gupta et al. the ability to expand this tissue to a clinical useful number has remained an important limitation [7]. The culture system reported here provides one method to expand epithelium in vitro prior to tissue engineering applications. Additionally, enteroids may be stored for later usage e.g. autologous transplantation. Lastly, the robust and reproducible nature of crypt culture represents an in vitro system to begin examination of normal homeostasis, response of the intestine to stress, and the potential for ISC dysregulation implicated in intestinal disease and cancer.

\section{Acknowledgments}

The authors wish to thank Dr. Joe Galanko in the UNC Center for Gastrointestinal Biology and Disease for assistance with statistical analysis.

Grants: This work was supported by the National Institutes of Health Grants R01-DK083325 (MAH), P30 DK034987 (SJH, MAH), and U01-DK085547 (SJH, MAH). The latter grant is part of the Intestinal Stem Cell Consortium, a collaborative research project funded by the National Institute of Diabetes and Digestive and Kidney Diseases and the National Institute of Allergy and Infectious Diseases. Additionally, this work was supported by the UNC Department of Surgery (MKF).

\section{References}

1. Buchman AL. Etiology and initial management of short bowel syndrome. Gastroenterology. 2006; 130:S5. [PubMed: 16473072]

2. Goulet $\mathrm{O}$, Baglin-Gobet S, Talbotec C, et al. Outcome and Long-Term Growth After Extensive Small Bowel Resection in the Neonatal Period: A Survey of 87 Children. Eur J Pediatr Surg. 2005; 15:95. [PubMed: 15877257]

3. Jeppesen PB, Mortensen PB. Enhancing Bowel Adaptation in Short Bowel Syndrome. Current gastroenterology reports. 2002; 4:338. [PubMed: 12149180]

4. Grikscheit TC, Siddique A, Ochoa ER, et al. Tissue-engineered small intestine improves recovery after massive small bowel resection. Ann Surg. 2004; 240:748. [PubMed: 15492554]

5. Agopian VG, Chen DC, Avansino JR, Stelzner M. Intestinal stem cell organoid transplantation generates neomucosa in dogs. J Gastrointest Surg. 2009; 13:971. [PubMed: 19165549]

6. Sala FG, Kunisaki SM, Ochoa ER, Vacanti J, Grikscheit TC. Tissue-Engineered Small Intestine and Stomach Form from Autologous Tissue in a Preclinical Large Animal Model. Journal of Surgical Research. 2009; 156:205. [PubMed: 19665143]

7. Gupta A, Dixit A, Sales KM, Winslet MC, Seifalian AM. Tissue engineering of small intestine-current status. Biomacromolecules. 2006; 7:2701. [PubMed: 17025341]

8. Bjerknes M, Cheng H. Intestinal epithelial stem cells and progenitors. Methods in Enzymology. 2006; 419:337. [PubMed: 17141062]

9. Ootani A, Li X, Sangiorgi E, et al. Sustained in vitro intestinal epithelial culture within a Wntdependent stem cell niche. Nat.Med. 2009; 15:701. [PubMed: 19398967]

10. Sato T, Vries RG, Snippert HJ, et al. Single Lgr5 stem cells build crypt-villus structures in vitro without a mesenchymal niche. Nature. 2009; 459:262. [PubMed: 19329995]

11. Sato T, Stange DE, Ferrante M, et al. Long-term expansion of epithelial organoids from human colon, adenoma, adenocarcinoma, and Barrett's epithelium. Gastroenterology. 2011; 141:1762. [PubMed: 21889923]

12. Jung P, Sato T, Merlos-Suarez A, et al. Isolation and in vitro expansion of human colonic stem cells. Nature Medicine. 2011; 17:1225.

13. Lahar N, Lei NY, Wang J, et al. Intestinal Subepithelial Myofibroblasts Support in vitro and in vivo Growth of Human Small Intestinal Epithelium. PLoS One. 2011; 6:1. 
14. Sato T, Vries RG, Snippert HJ, et al. Single Lgr5 stem cells build crypt-villus structures in vitro without a mesenchymal niche. Nature. 2009; 459:262. [PubMed: 19329995]

15. Wright NA. Epithelial stem cell repertoire in the gut: clues to the origin of cell lineages, proliferative units and cancer. Int.J Exp.Pathol. 2000; 81:117. [PubMed: 10762441]

16. Henning, SJ.; Rubin, DC.; Shulman, RJ. Ontogeny of the intestinal mucosa.. In: Johnson, LR., editor. Physiology of the Gastrointestinal Tract. Raven Press; New York: 1994. p. 571-610. 


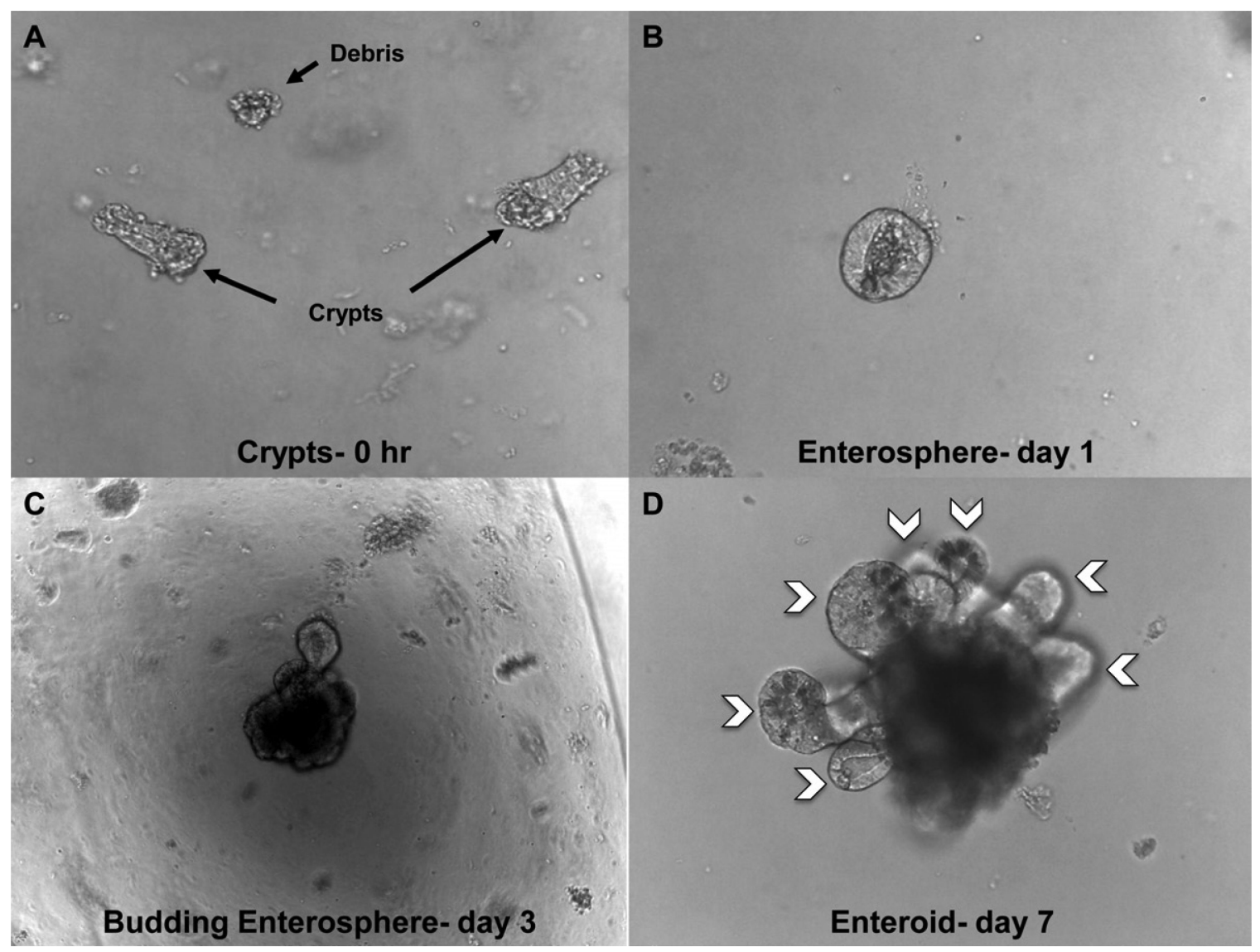

FIG. 1. Images demonstrating progression of crypts in culture

All panels are at 200x; (A) Crypts on initial plating for goal density of 25 crypts/well (B) Representative enterosphere at 1 day; (C) Enterosphere budding at 3 days; and D) Representative enteroid at 7 days, note crypt buds (arrowhead $\wedge$ ) are roughly the same size as initially plated crypts. 

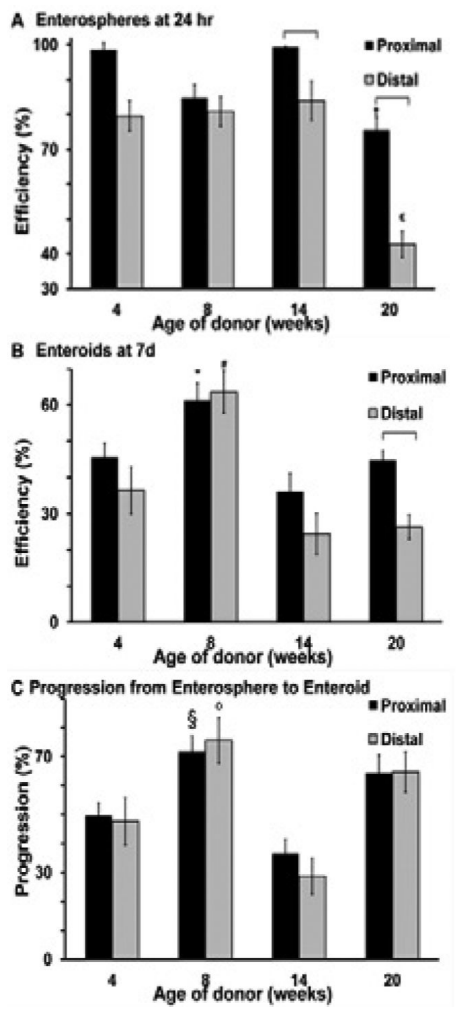

FIG. 2. Quantitative behavior of crypts in culture

(A) Enterosphere efficiency. Percent of crypts surviving as enteroids at $24 \mathrm{hr}$, measured by: region of small intestine and age of animal. Symbols represent $\mathrm{p}<0.05$ for: $\Pi=$ between proximal and distal, $\dagger=$ proximal $20<14 \mathrm{wk}, €=$ distal $20<4,8,14 \mathrm{wks}$. (B) Enteroid efficiency. Percent of crypts yielding enteroids at 7 days, measured by: region of small intestine and age of animal. Symbols represent $\mathrm{p}<0.05$ for: $\Pi=$ between proximal and distal, * = proximal $8 \mathrm{wk}>14,20 \mathrm{wk}, \#=$ distal $8 \mathrm{wk}>4,14,20 \mathrm{wk}(C)$ Enterosphere progression. Percent of enterospheres at $24 \mathrm{hr}$ progressing to enteroids at 7 days, measured by: region of small intestine and age of animal. Symbols represent $p<0.05$ for: $\S=$ proximal $8>4,14 \mathrm{wk},{ }^{\circ}=$ distal $8>14 \mathrm{wk}$.

Data are shown as means \pm SE for biologic $n=4,6$, 4, and 6 at ages 4, 8, 12, and $20 \mathrm{wk}$ respectively. For simplicity of graphical visualization the middle region has been omitted from figs $2 \mathrm{~A}-\mathrm{C}$. 


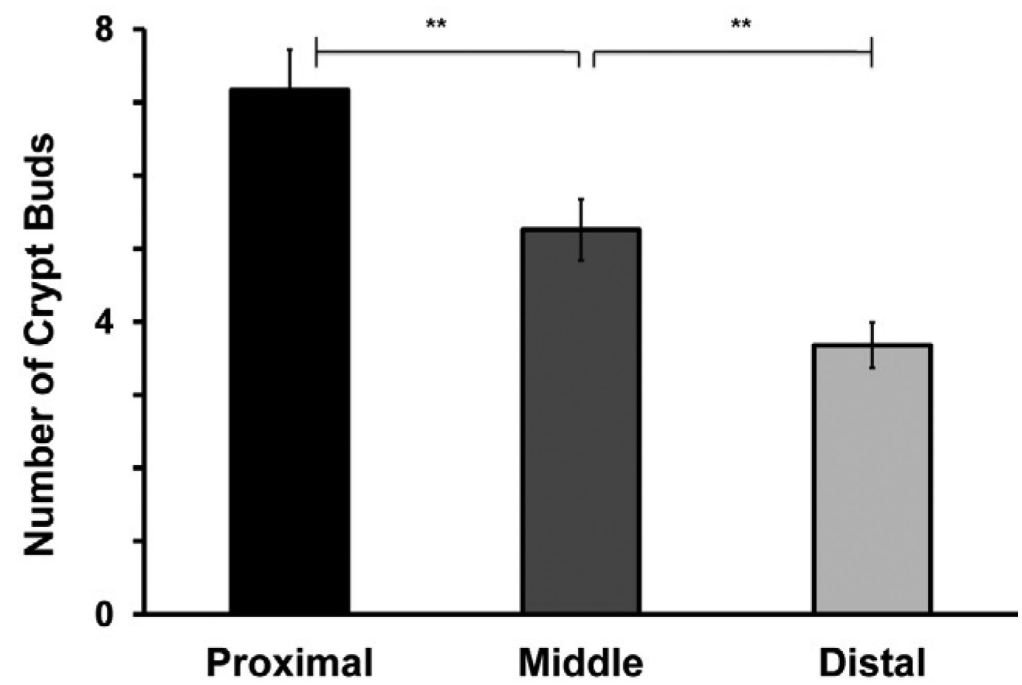

FIG. 3. Crypt buds by region

Number of new crypt domains (buds) per enteroid at 7 days measured by: region of small intestine (proximal, middle, or distal) pooled for all ages. All data are represented as means \pm SE with biologic $\mathrm{n}=20 .(* * \mathrm{p}<0.001)$ 


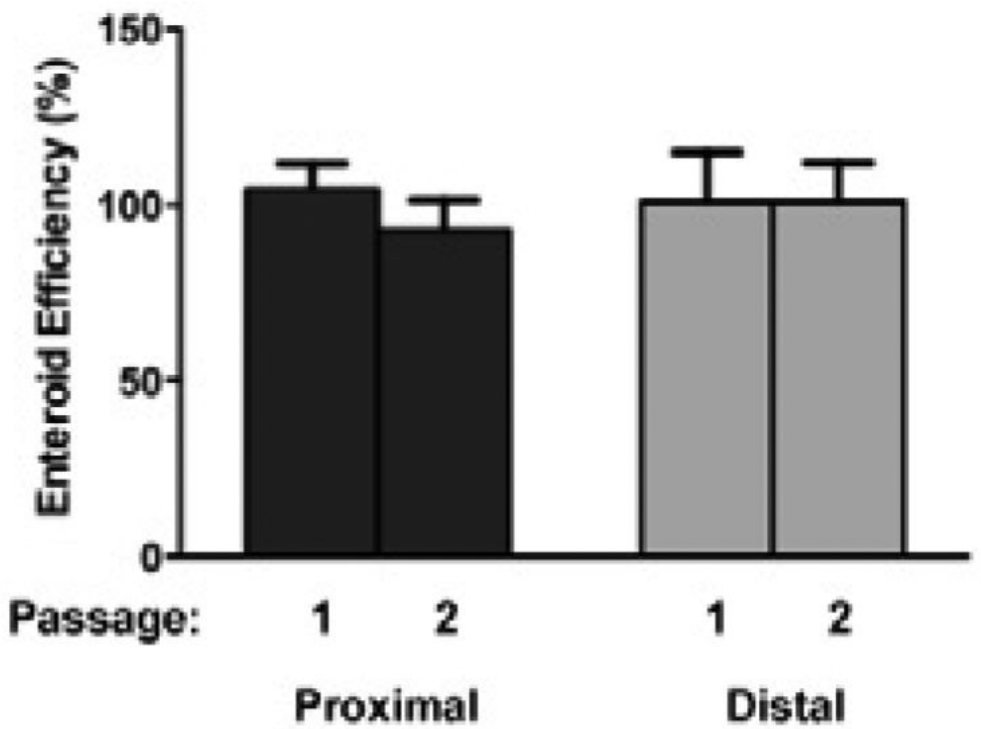

FIG. 4. Continued crypt expansion

Enteroid efficiency of the proximal and distal intestine after passage 1 (passaged at day 7, quantified at day 14) and passage 2 (passaged at day 14, quantified at day 21). All data are shown as means \pm SE biologic $n=3$. 


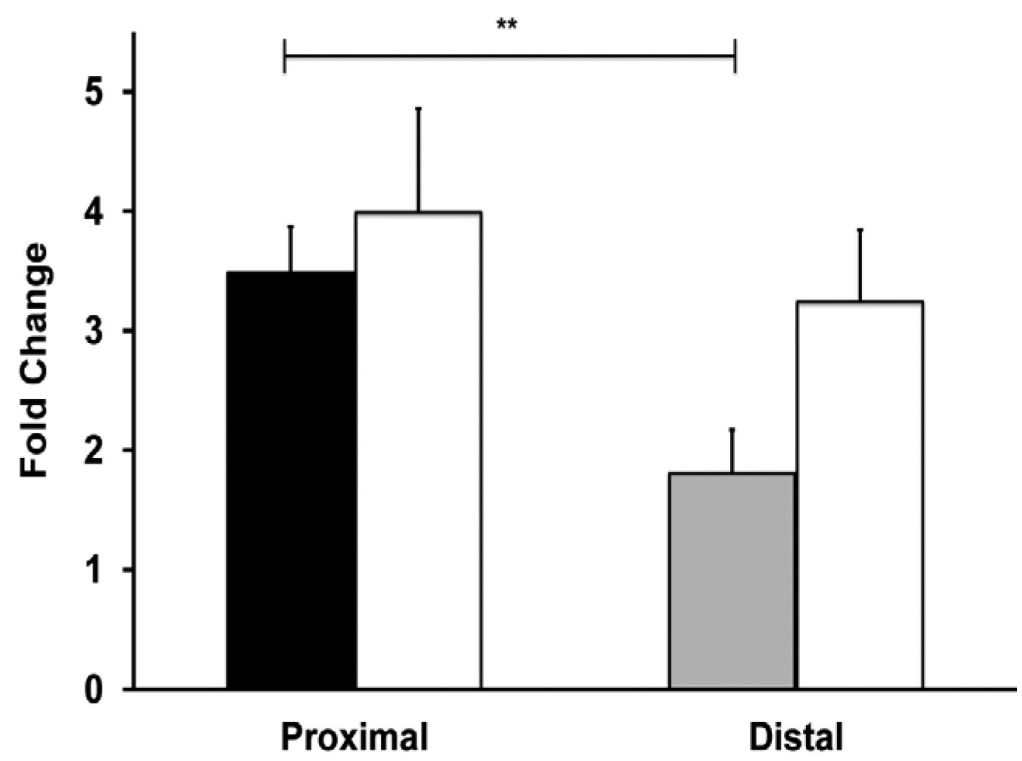

FIG. 5. Crypt expansion by region

Fold expansion of crypts at 7 days measured by region (proximal or distal) and pooled for all ages (black and gray bars respectively) biologic $\mathrm{n}=20$ for all regions. $(* * \mathrm{p}<0.001)$ Fold expansion for the single best age $(8 \mathrm{wks})$ is represented by the open bars, $n=6$. All data are represented as means $\pm \mathrm{SE}$ 